\title{
The exotic chemical composition of the Sagittarius dwarf spheroidal galaxy
}

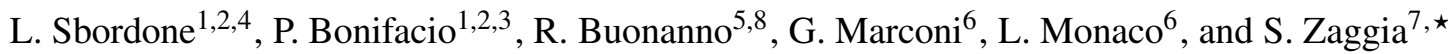 \\ 1 CIFIST Marie Curie Excellence Team, France \\ 2 Observatoire de Paris, GEPI, 5 place Jules Janssen, 92195 Meudon, France \\ e-mail: luca.sbordone@obspm. fr \\ INAF - Osservatorio Astronomico di Trieste, via G. B. Tiepolo 11, 34131 Trieste, Italy \\ 4 INAF - Osservatorio Astronomico di Roma, via di Frascati 33, 00040 Monte Porzio Catone, Rome, Italy \\ 5 Università di Roma "Tor Vergata”, via della Ricerca Scientifica 1, 00133 Rome, Italy \\ ${ }^{6}$ ESO - European Southern Observatory, Alonso de Cordova, 3107 Santiago, Chile \\ 7 INAF - Osservatorio Astronomico di Padova, Vicolo dell'Osservatorio 5, 35122 Padua, Italy \\ 8 ASI Science Data Center, via Galileo Galilei, 00044 Frascati, Italy
}

Received 12 September 2006 / Accepted 29 November 2006

\begin{abstract}
Context. The Sagittarius dwarf spheroidal galaxy is the nearest neighbor of the Milky Way. Moving along a short period quasi-polar orbit within the Halo, it is being destroyed by the tidal interaction with our Galaxy, losing its stellar content along a huge stellar stream.

Aims. We study the detailed chemical composition of 12 giant stars in the Sagittarius dwarf Spheroidal main body, together with 5 more in the associated globular cluster Terzan 7, by means of high resolution VLT-UVES spectra.

Methods. Abundances are derived for up to 21 elements from $\mathrm{O}$ to $\mathrm{Nd}$, by fitting lines $E W$ or line profiles against ATLAS 9 model atmospheres and SYNTHE spectral syntheses calculated ad-hoc. Temperatures are derived from $(V-I)_{0}$ or $(B-V)_{0}$ colors and gravities from $\mathrm{Fe}$ I - Fe II ionization equilibrium.

Results. The metallicity of the observed stars is between $[\mathrm{Fe} / \mathrm{H}]=-0.9$ and 0 . We detected a highly peculiar "chemical signature", with undersolar $\alpha$ elements, $\mathrm{Na}, \mathrm{Al}, \mathrm{Sc}, \mathrm{V}, \mathrm{Co}, \mathrm{Ni}, \mathrm{Cu}$, and $\mathrm{Zn}$, among others, and overabundant $\mathrm{La}, \mathrm{Ce}$, and $\mathrm{Nd}$. Many of these abundance ratios (in particular light-odd elements and iron peak ones) are strongly at odds with what is observed within the Milky Way, so they may be a very useful tool for recognizing populations originating within the Sagittarius dwarf. This can be clearly seen in the case of the globular Palomar 12, which is believed to have been stripped from Sagittarius: the cluster shows precisely the same chemical "oddities", thus finally confirming its extragalactic origin.
\end{abstract}

Key words. stars: abundances - stars: atmospheres - Galaxy: abundances - galaxies: dwarf - galaxies: individual: Sgr dSph Galaxy: globular clusters: individual: Terzan 7

\section{Introduction}

Dwarf spheroidal galaxies ( $\mathrm{dSph}$ ) have in recent years become a highly popular subject of investigation. The interest in these objects has been largely driven by the key role they are supposed to play in the buildup process of larger galaxies such as the Milky Way. The available detailed abundance ratios for stars in Local Group (LG) dSph nevertheless show that present day $\mathrm{dSph}$ are undesirable candidates for hierarchical merging building blocks (see Vladilo et al. 2003; Venn et al. 2004, and references therein). The distinctive abundance ratios (most notably, the low $[\alpha / \mathrm{Fe}]$ ratio) observed in the LG dSph hint at star formation histories that are remarkably different from the one characteristic of the MW. This makes it difficult for evolved dSphs to have played a significant role in building up our Galaxy. Such

* Tables A1-A3 are only available in electronic form at the CDS via anonymous ftp to cdsarc.u-strasbg.fr (130.79.128.5) or via

http://cdsweb.u-strasbg.fr/cgi-bin/qcat?J/A+A/465/815 findings are, after all, not surprising: undisturbed, low-mass $\mathrm{dSph}$ constitute a different environment from the MW, where low star formation rates and highly efficient galactic winds have likely played a major role (Lanfranchi \& Matteucci 2003, 2004; Lanfranchi et al. 2006a). Dwarf spheroidals are nevertheless not ruled out as MW "building blocks", but the main merging phase should have taken place at a very early stage, allowing the subsequent evolution to differentiate between the surviving $\mathrm{dSph}$ and the larger, merged structures.

At least one major merging episode is currently taking place in the MW, at the expense of the Sagittarius dSph (Sgr dSph, Ibata et al. 1994, 1995). The nearest known dSph (26.3 Kpc, Monaco et al. 2004), Sgr dSph, is being tidally destroyed while moving along its quasi-polar, short period (less than $1 \mathrm{GYr}$ ) orbit around the MW (Ibata et al. 1997; Helmi \& White 1999), and its stars are dispersing along a huge stream in the Halo (Majewski et al. 2003; Belokurov et al. 2006).

In previous works (Bonifacio et al. 2000, 2004; Sbordone et al. 2005, henceforth Papers I, II, and III, respectively), 
we presented the chemical abundances for a total of 12 stars in the Sgr dSph main body and in the associated globular cluster Terzan 7. Paper I reported abundances for 20 elements from $\mathrm{O}$ to Eu in two Sgr dSph main body stars, Paper II added Fe and $\alpha$ elements for 10 more stars, while Paper III analyzed Fe, $\alpha$ elements, and $\mathrm{Ni}$ in 5 giants in Terzan 7. In two more papers, we also obtained iron and $\alpha$ elements abundances for 15 brighter RGB stars of Sgr (Monaco et al. 2005a), and sulfur abundances for three stars in Ter 7 (Caffau et al. 2005). The present paper unifies, extends, and revisits the results presented in Papers I, II, and III:

- the temperature scale has been recalibrated for the 12 Sgr dSph main body stars by using photometries and reddening from Monaco et al. (2002). This has been made to homogenize the temperature scale with the one used in Monaco et al. (2005a). See Sect. 2 for details;

- the array of abundances has been extended to up to 21 species including $\mathrm{O}, \mathrm{Na}, \mathrm{Mg}, \mathrm{Al}, \mathrm{Si}, \mathrm{Ca}, \mathrm{Sc}, \mathrm{Ti}, \mathrm{V}, \mathrm{Cr}$, $\mathrm{Mn}, \mathrm{Fe}, \mathrm{Co}, \mathrm{Ni}, \mathrm{Cu}, \mathrm{Zn}, \mathrm{Y}, \mathrm{Ba}, \mathrm{La}, \mathrm{Ce}$, and Nd;

- with respect to the previous papers, updated atomic data are used for $\mathrm{Mg}, \mathrm{Ca}, \mathrm{La}, \mathrm{Ce}$, and $\mathrm{Nd}$.

\section{Observations, data reduction, and analysis}

As stated above, the present work uses the same data as was employed in Papers I and II for the Sgr dSph main body and in Paper III for Terzan 7 stars. The reader is thus referred there for the details of the observations. Table 1 lists photometry and derived atmospheric parameters for the $12 \mathrm{Sgr} \mathrm{dSph}$ main body and the 5 Ter 7 giants. Coordinates can be found in Paper II (Sgr dSph main body) and Paper III (Ter 7). A sample of the spectra for the 12 Sgr dSph stars is shown in Fig. 1 of Paper II and in Fig. 1 of Paper III for the 5 Ter 7 giants. The temperature scale was recalibrated in the main body of Sgr dSph due to the adoption of Monaco et al. (2002) photometry and Layden \& Sarajedini (2000) reddening, while we used Marconi et al. (1998) photometry and reddening estimates in Papers I and II. This change came from the need to use a temperature scale that is both homogeneous across our present work and applicable to future studies, too. Moreover Monaco et al. (2002) photometry covers a much larger field and it was used as a basis for FLAMES candidates selection (see Zaggia et al. 2004).

Effective temperatures were derived from dereddened $(V-I)$ colors by means of the Alonso et al. $(1999,2001)$ calibration for giant stars. The change in the photometry and reddening correction $E(V-I)=0.22$ in Marconi et al. (1998) were replaced by $E(V-I)=0.18$ from Layden \& Sarajedini $(2000)^{1}$. This led to significantly lower derived effective temperatures, with a mean decrease of about $250 \mathrm{~K}$. The reason for such a change comes partly from the different reddening estimates ( 0.04 difference in $V-I$ color), but is mainly due to an offset between the two photometries (0.074 mean). As a further test, we checked the temperatures derived from 2MASS $J-K$ colors for stars in this work and in Monaco et al. (2005a). Nevertheless, the Sgr dSph stars presented here are generally too faint for 2MASS to provide reliable colors for them, and the derived temperatures were scattered over more than $1000 \mathrm{~K}$. For the Monaco et al. (2005a) stars, 2MASS $J-K$ colors led to temperatures $75 \mathrm{~K}$ hotter on average than the ones based on Monaco et al. (2002)

\footnotetext{
1 Reddening estimate from Schlegel et al. (1998) is very near to the Layden \& Sarajedini (2000) one, with a typical value of $E(V-I)=0.19$ for our Sgr dSph main body stars.
}

Table 1. Photometry and atmospheric parameters for the studied stars. Employed colors are $(V-I)_{0}$ for the main body of Sgr dSph and $(B-V)_{0}$ for Ter 7 .

\begin{tabular}{llllll}
\hline \hline Star & $\begin{array}{l}V \\
\text { mag }\end{array}$ & $\begin{array}{l}(V-I)_{0} \\
\text { mag }\end{array}$ & $\begin{array}{l}T_{\text {eff }} \\
\mathrm{K}\end{array}$ & $\begin{array}{l}\log g \\
\operatorname{cgs}\end{array}$ & $\begin{array}{l}\xi \\
\mathrm{km} \mathrm{s}^{-1}\end{array}$ \\
\hline Sgr 432 & 17.700 & 1.013 & 4713 & 2.2 & 1.20 \\
Sgr 628 & 18.228 & 1.040 & 4656 & 2.1 & 1.75 \\
Sgr 635 & 18.186 & 1.074 & 4588 & 2.1 & 1.50 \\
Sgr 656 & 18.217 & 1.008 & 4723 & 2.1 & 1.50 \\
Sgr 709 & 18.260 & 1.034 & 4669 & 2.2 & 1.20 \\
Sgr 716 & 18.282 & 1.012 & 4715 & 2.1 & 1.60 \\
Sgr 717 & 18.282 & 1.007 & 4726 & 2.0 & 1.10 \\
Sgr 772 & 18.392 & 1.070 & 4596 & 1.9 & 1.60 \\
Sgr 867 & 18.465 & 1.031 & 4675 & 1.7 & 1.95 \\
Sgr 879 & 18.516 & 1.073 & 4590 & 1.9 & 1.30 \\
Sgr 894 & 18.507 & 1.067 & 4602 & 2.1 & 1.50 \\
Sgr 927 & 18.580 & 1.079 & 4578 & 2.1 & 1.30 \\
& & $(B-V)_{0}$ & & & \\
Ter7 1272 & 16.62 & 1.15 & 4421 & 1.2 & 1.45 \\
Ter7 1282 & 16.08 & 1.30 & 4203 & 1.3 & 1.60 \\
Ter7 1515 & 16.76 & 1.12 & 4468 & 2.0 & 1.45 \\
Ter7 1665 & 15.04 & 1.50 & 3945 & 0.8 & 1.60 \\
Ter7 1708 & 16.08 & 1.28 & 4231 & 1.2 & 1.70 \\
\hline
\end{tabular}

$(V-I)$ colors, while on Ter 7 there was no systematic discrepancy between the two temperature scales. We will show Later on (see Sect. 3.3) how the changes in the physical parameters of the atmospheres do not alter significantly the "scientific output" of this research.

Similar to what we did in Papers I through III, onedimensional, LTE atmosphere models were computed for the observed stars by means of our GNU-Linux ported version of the ATLAS code. ATLAS 9 (using opacity distribution functions, henceforth ODF) models were used for all the stars except for star \#1665 in Terzan 7, which required an ATLAS 12 (opacity sampling) model due to its very low temperature and gravity (see Sbordone et al. 2005, for details). For the ODF-based models, no $\alpha$-enhancement was assumed (since $[\alpha / \mathrm{Fe}]$ appears to be solar or sub-solar); instead, "new" type ODF (Castelli \& Kurucz 2003) were used. Abundances were computed from measured line equivalent widths $(E W)$ by means of WIDTH, and the model gravity was set by imposing the Fe I-Fe II ionization equilibrium. The microturbulence was then set by requesting abundances of Fe I lines to be independent of the line $E W$. Abundances for lines affected by strong hyperfine splitting, or for which $E W$ measurement was problematic (e.g. the $630 \mathrm{~nm}$ OI line), were derived by spectral synthesis, using SYNTHE (for ATLAS, WIDTH, and SYNTHE see Kurucz 1993, 2005; Sbordone et al. 2004; Sbordone 2005).

To produce the same Fe II line strength with a lower $T_{\text {eff }}$, lower gravity is needed. Our gravity estimates decreased by about $0.3-0.4$ dex with respect to the ones in Paper II, leading to values of $\log g \sim 2.1$. This caused the compatibility between isochrone and ionization gravities to worsen somewhat in comparison with Papers I and II: superimposing Girardi et al. (2002) isochrones of compatible age and metallicity now leads to deriving typical gravities on the order of $\log g \sim 2.4$, for a mean discrepancy of about 0.35 dex.

Complete line lists, with employed $\log g f$ values, measured $E W$ and derived abundances are available in the online version. An excerpt is available in Table 2. In these tables, Na line-byline abundances are listed without NLTE corrections; for lines that have been synthesized, "syn" substitutes the EW. Papers I and II employed the same set of lines and atomic data (with the 


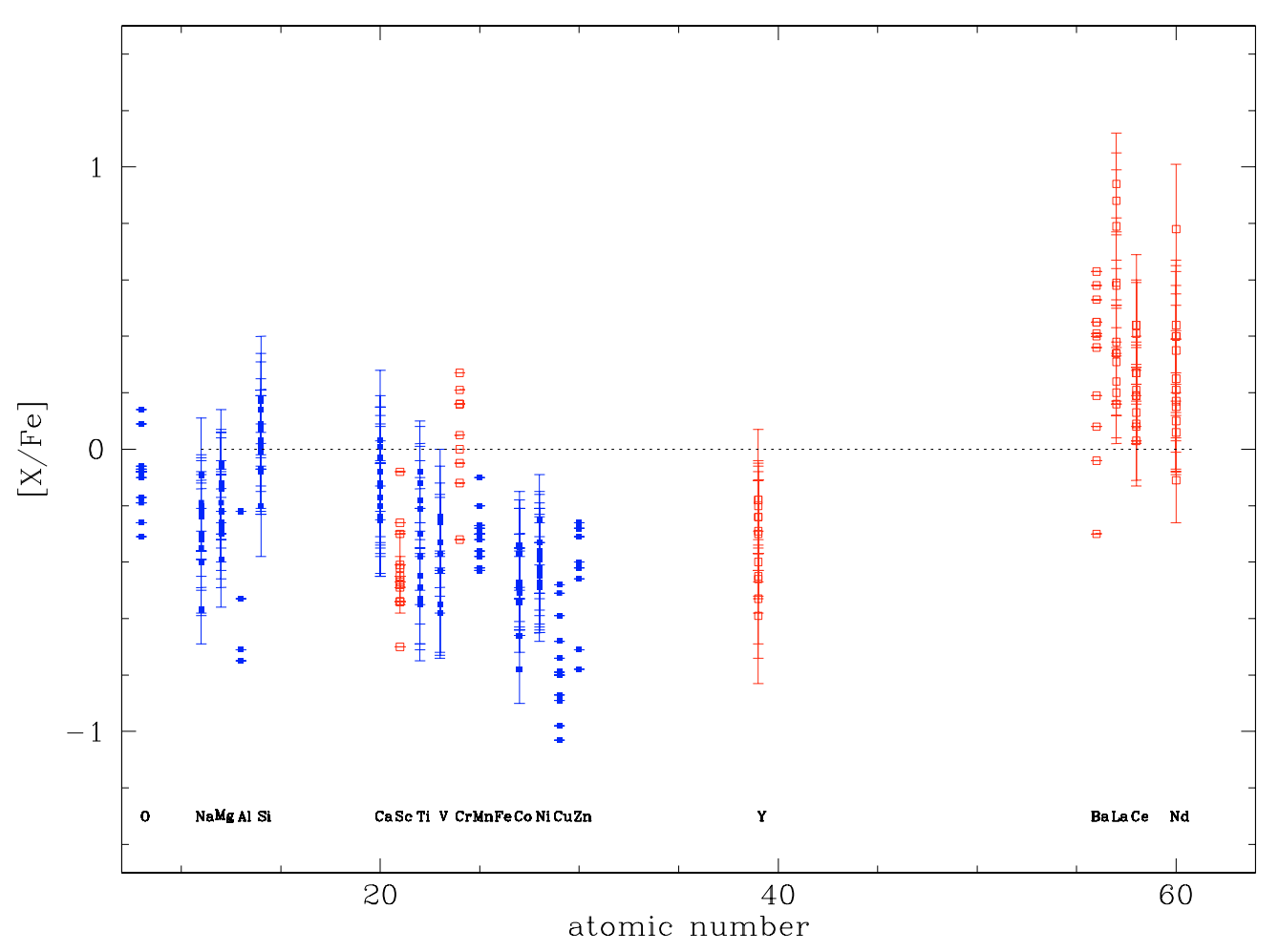

Fig. 1. The "chemical signature" of the $12 \mathrm{Sgr}$ dSph stars: $[\mathrm{X} / \mathrm{Fe}]$ ratios are plotted against atomic number. The dashed line at 0 value represents the solar abundance ratios. As in Tables 4-6, ratios are against Fe I for neutral species (filled squares), against Fe II for the ionized ones (Sc, Cr, Y, $\mathrm{Ba}, \mathrm{La}, \mathrm{Ce}, \mathrm{Nd}$, open squares). Error bars are the same listed in Tables 4-6, so that species measured on a single line do not show any error bar. Large departures from solar ratios can be seen throughout all the sampled elements: see text for details.

exception of O I $630 \mathrm{~nm}$ line) for the elements which were common among the two. Paper III used the same set of lines of Papers I and II for the Dichroic I spectra and (obviously) a different one for the Dichroic II spectra. Nevertheless, in Paper III $\log g f$ were updated for $\mathrm{Mg}$ and $\mathrm{Ca}$ lines. Globally, updates are as follows:

- O I we used the Storey \& Zeippen (2000) $\log g f$ already from Paper II on. The O I $630 \mathrm{~nm}$ line is measured by spectral synthesis. The line is heavily blended with a weak Ni I feature for which we used the Johansson et al. (2003) laboratory $\log g f$;

- Mg I from Paper III on, we use the Gratton et al. (2003) $\log g f$ where applicable;

- Si I solar Edvardsson et al. (1993) $\log g f$ values are used where applicable;

- Ca I since Paper III we have used, where present, Smith \& Raggett (1981) furnace $\log g f ; 586.7 \mathrm{~nm}$ line has Gratton et al. (2003) $\log g f$;

- Zn I we used the $\log g f$ values of Biemont \& Godefroid (1980), which provide a good agreement between the photospheric and the meteoritic $\mathrm{Zn}$ abundances;

- La II recent $\log g f$ values from Lawler et al. (2001) are used here when available;

- Ce II $\log g f$ values are taken from Hill et al. (1995);

- Nd II new $\log g f$ values are taken from Den Hartog et al. (2003).

All the other atomic data are the ones provided in the line lists included with our ATLAS suite port ${ }^{2}$, which come from R. L. Kurucz website ${ }^{3}$. Hyperfine splitting was used to synthesize lines for $\mathrm{Mn} \mathrm{I}, \mathrm{Co} \mathrm{I}$, and $\mathrm{Cu}$ I. Solar isotopic ratios were assumed.

\footnotetext{
2 Available at http://wwwuser.oat.ts.astro.it/atmos/

3 http://kurucz.harvard.edu/
}

Many of the employed $\mathrm{Na}$ and $\mathrm{Al}$ lines are known to be significantly affected by NLTE (Baumueller \& Gehren 1997; Baumueller et al. 1998; Gratton et al. 1999). The NLTE corrections are generally a function of metallicity, atmospheric parameters, and line strength, and unfortunately no correction computations have been produced for Al lines for giant stars such as the ones we are dealing with. As a consequence, we publish LTE abundances for Al. For Na lines, correction are computed for giant stars by Gratton et al. (1999). Their grid extends down to $\log (g)=1.5$ and $T_{\text {eff }}=4000 \mathrm{~K}$, which fits all the Sgr dSph main body stars and one of the Ter 7 stars. For these stars, we interpolated the correction values and present the corrected abundances here. The four other Ter 7 stars go beyond the grid in gravity or temperature, or both. In these cases, extrapolated values can be used, but given the complex behavior of NLTE corrections in phase space, we preferred to adopt a single value for $A_{\mathrm{NLTE}}-A_{\mathrm{LTE}}=0.2$.

\section{Results}

Absolute abundances for Sgr dSph stars, solar assumed abundances, $[\mathrm{Fe} / \mathrm{H}],[\mathrm{X} / \mathrm{Fe}]$, and associated errors are listed in Tables 4 and 5. The same data for Terzan 7 stars are in Table 6. In these tables, the upper panel indicates the assumed solar abundances and absolute measured abundances for the sample stars. The published error is simply the rms of the measured lines, thus is absent for species where a single line has been used. The lower panel lists $[\mathrm{X} / \mathrm{Fe} \mathrm{I}]$ ratios for the neutral species, and $[\mathrm{X} / \mathrm{Fe} \mathrm{II}] \mathrm{ra}-$ tios for the ionized ones. $[\mathrm{O} / \mathrm{Fe} \mathrm{II}]$ is listed instead of $[\mathrm{O} / \mathrm{Fe} \mathrm{I}]$ due to the strong sensitivity to gravity of [O I] $630.03 \mathrm{~nm}$ line. 
Table 2. An excerpt from the detailed line tables available in the Online version. Employed lines, $\log g f$ values, $\log g f$ sources, observed $E W$ and derived abundances for Sgr dSph stars \#432 to \#716. LTE abundanced are listed here for the Na lines.

\begin{tabular}{|c|c|c|c|c|c|c|c|c|c|c|c|c|c|c|c|}
\hline Ion & $\begin{array}{r}\lambda \\
(\mathrm{nm})\end{array}$ & $\log g f$ & $\begin{array}{l}\text { Source of } \\
\log g f^{a}\end{array}$ & $\begin{array}{r}E W \\
(\mathrm{pm}) \\
432\end{array}$ & $A(\mathrm{X})$ & $\begin{array}{r}E W \\
(\mathrm{pm}) \\
628\end{array}$ & $A(\mathrm{X})$ & $\begin{array}{r}E W \\
(\mathrm{pm}) \\
635\end{array}$ & $A(\mathrm{X})$ & $\begin{array}{r}E W \\
(\mathrm{pm}) \\
656\end{array}$ & $A(\mathrm{X})$ & $\begin{array}{r}E W \\
(\mathrm{pm}) \\
709\end{array}$ & $A(\mathrm{X})$ & $\begin{array}{r}E W \\
(\mathrm{pm}) \\
716\end{array}$ & $A(\mathrm{X})$ \\
\hline $\mathrm{O}_{\mathrm{I}}$ & 630.0304 & 9717 & STZ & syn & 8.03 & syn & 8.35 & syn & 8.33 & syn & 8.15 & syn & 8.50 & syn & 8.54 \\
\hline $\mathrm{NaI}$ & 8.2633 & 0700 & KP & - & - & 10.67 & 5.79 & 7.56 & 5.33 & 9.61 & 5.76 & 11.23 & 6.06 & 11.18 & 5.94 \\
\hline $\mathrm{Na}$ & 615.4227 & -1.560 & $\mathrm{KP}$ & - & - & 4.38 & 5.72 & 1.95 & 5.21 & 3.36 & 5.62 & - & - & 2.47 & 5.44 \\
\hline $\mathrm{Na}$ & 616.0747 & -1.260 & KP & - & - & 5.26 & 5.56 & 3.60 & 5.26 & 4.34 & 5.49 & 6.07 & 5.74 & 5.77 & 5.69 \\
\hline
\end{tabular}

${ }^{a}$ Codes for the source of the $\log g f$ can be found in the bibliography.

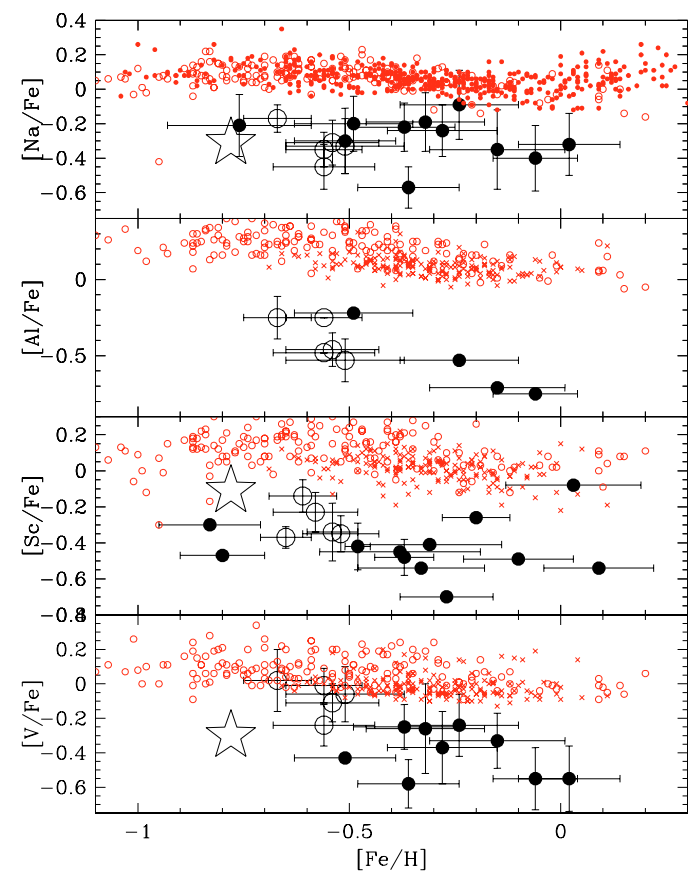

Fig. 2. $[\mathrm{Na} / \mathrm{Fe}],[\mathrm{Al} / \mathrm{Fe}],[\mathrm{Sc} / \mathrm{Fe}]$, and $[\mathrm{V} / \mathrm{Fe}]$ plotted against $[\mathrm{Fe} / \mathrm{H}]$. The symbols are as follows: large filled circles, Sgr dSph main body; large open circles Ter 7; large star, Palomar 12 (mean value for the stars in Cohen 2004); small open circles, MW sample from Reddy et al. (2006); small crosses, MW sample from Reddy et al. (2003); small filled circles, MW sample from Venn et al. (2004), which also includes Reddy et al. (2003) stars. In the Na plot, the value for Pal 12 has been corrected by the same amount as was used for low-gravity Ter 7 stars $\left(A_{\mathrm{NLTE}}-\right.$ $A_{\mathrm{LTE}}=0.2$ ).

\subsection{The Sgr dSph main body}

As already stated in Papers I and II, the 12 Sgr dSph stars have a relatively high mean metallicity $([\mathrm{Fe} / \mathrm{H}]$ between -0.89 and 0.02): the average value is $[\mathrm{Fe} / \mathrm{H}]=-0.36$. In comparison with Papers I and II, the variaton in the atmospheric parameters led to a slight metallicity decrease.

A full picture of the "chemical signature" of Sgr dSph main body is presented in Fig. 1. At first glance, Sgr dSph appears to bear the signs of a highly peculiar chemical evolution. We thus now treat the various element groups in some detail.

The $\alpha$ elements show the same behavior as described in Papers I and II (Fig. 3). The $\alpha$ elements show solar or undersolar ratios against iron, with a significant trend with the metallicity, leading $\mathrm{Mg}$ and $\mathrm{Ca}$ in particular to reach heavy underabundances in the most metal-rich stars of the sample $([\mathrm{Mg} / \mathrm{Fe}]=-0.39$ in star \#709). Silicon instead remains nearer to solar values, and even shows some enhancement in some cases $([\mathrm{Si} / \mathrm{Fe}]=0.18$ in star \#894). Although its inclusion among $\alpha$ elements is not totally correct from a nucleosynthetic point of view (see Burbidge et al. 1957), Titanium also shows heavy underabundance compared to solar values.

Light odd-atomic number elements $\mathrm{Na}, \mathrm{Al}, \mathrm{Sc}$, and $\mathrm{V}$ also show a highly significant underabundance with respect to solar values (see Fig. 2). A hint of a trend with metallicity can be seen in the $[\mathrm{Al} / \mathrm{Fe}]$ graph, but since a single line was used in Sgr dSph main body stars, and considering the possible presence of NLTE effects, this cannot be considered significant.

Iron-peak elements $\mathrm{Co}, \mathrm{Ni}, \mathrm{Cu}$, and $\mathrm{Zn}$ (Fig. 4) perhaps display the most intriguing anomalies, showing constantly undersolar ratios with no clear metallicity trends. Although both $\mathrm{Cu}$ and $\mathrm{Zn}$ have been measured using a single line, these are rather strong and clear transitions whose fit is robust.

Finally, heavy n-capture elements Y, Ba, La, Ce and $\mathrm{Nd}$ (Y, $\mathrm{Ba}, \mathrm{La}$, and $\mathrm{Nd}$ in Fig. 5) also show interesting patterns. There, $\mathrm{Y}$ appears to be undersolar at low metallicity, with a slight increasing trend with metallicity that leads to solar $[\mathrm{Y} / \mathrm{Fe}] \mathrm{ra}-$ tios around solar $[\mathrm{Fe} / \mathrm{H}]$. Ba is oversolar with a moderately increasing trend with metallicity and generally outside the range of $[\mathrm{Ba} / \mathrm{Fe}]$ variation within the MW, but three Sgr dSph main body stars (\#635, \#716, and \#867) show MW-like [Ba/Fe] ratios. Spectra inspection did not show clear anomalies in the $\mathrm{Ba}$ lines in these stars, but since we are using a single Ba II feature, the hypothesis of some contamination of the line cannot be ruled out. Lantanium abundances show an above-average spread, but $[\mathrm{La} / \mathrm{Fe}]$ appears to be consistently oversolar and increasing with metallicity, reaching rather extreme values at solar metallicity $([\mathrm{La} / \mathrm{Fe}]=0.94$ at $[\mathrm{Fe} / \mathrm{H}]=0.02$ in star \#709). Finally, $\mathrm{Nd}$ starts from MW-like (essentially solar) ratios at low metallicity, then increasing slightly but never truly standing out with respect to the values observed within the MW.

\subsection{Terzan 7}

In Figs. 3 through 5 Ter 7 stars are always represented by large open circles. As can be seen, Ter 7 chemical composition appears to closely match the one observed in the Sgr dSph main body at corresponding metallicity. Nevertheless some differences can be seen: ratios against iron are slightly above the Sgr dSph values for $\mathrm{V}, \mathrm{Co}, \mathrm{Ni}, \mathrm{Y}$, and $\mathrm{Nd}$. This is probably due to the lower reliability of the analysis in such low gravity - low temperature atmospheres.

\subsection{Error budget}

In Table 3 we report the variations in abundances, $[\mathrm{X} / \mathrm{Fe}]$ and $[\mathrm{Fe} / \mathrm{H}]$ for star \#656, as due to the variation in atmospheric parameters between Papers I/II and this work. Absolute abundances from Paper II and this work are also listed to ease the 


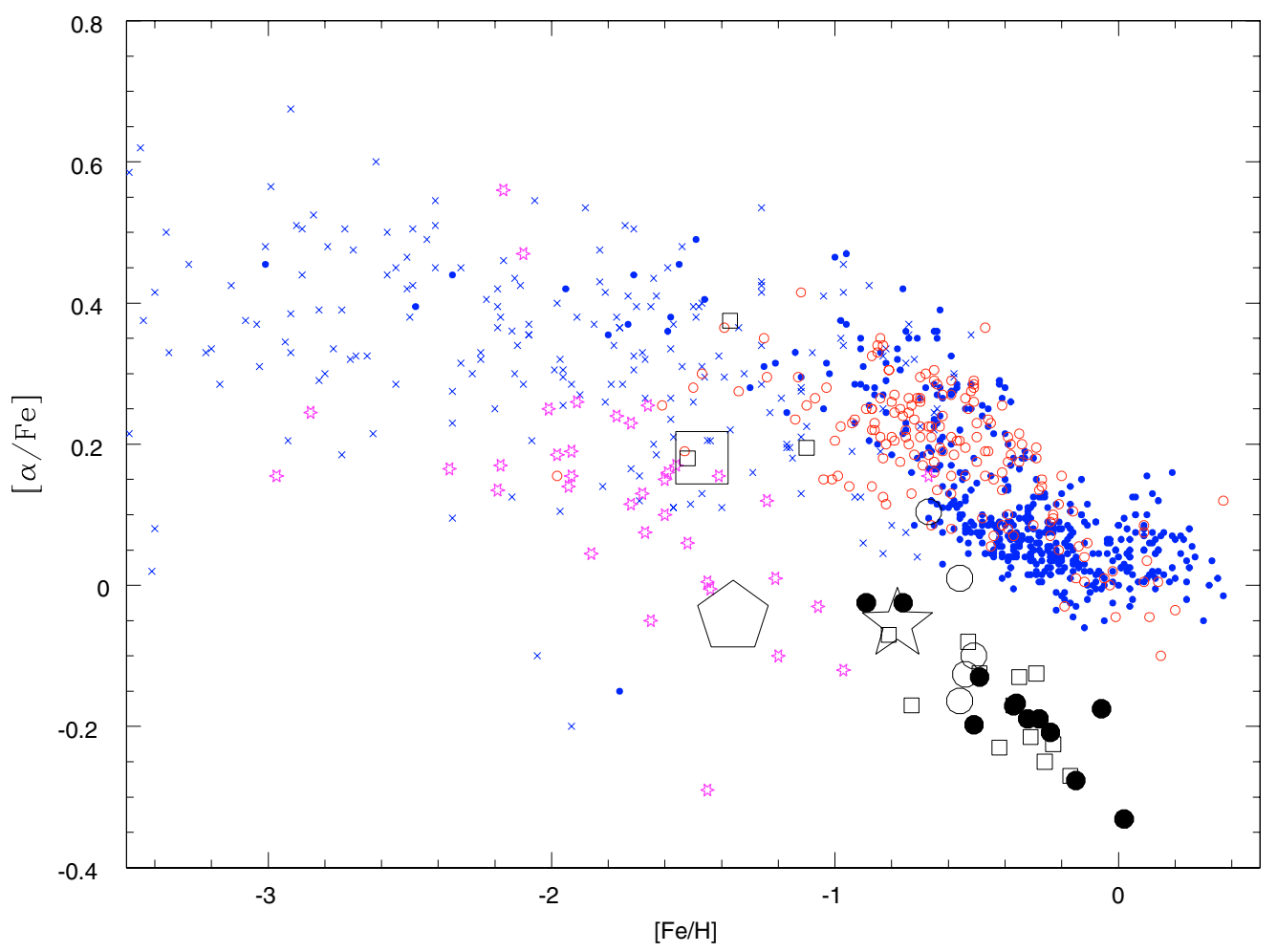

Fig. 3. $[\alpha / \mathrm{Fe}]$ (defined as mean of $[\mathrm{Mg} / \mathrm{Fe}]$ and $[\mathrm{Ca} / \mathrm{Fe}]$ ) is plotted against $[\mathrm{Fe} / \mathrm{H}]$ for various samples: large filled dots, Sgr dSph main body (this work); large open dots, Ter 7 (this work); open squares, Sgr dSph main body upper RGB stars (Monaco et al. 2005a); small open stars, LG dSph stars (Car, Dra, For, Leo I, Scl, Sex, UMi, Shetrone et al. 2001, 2003). Small symbols refer to MW samples: filled circles, Venn et al. (2004) thin and thick disk stars; crosses, Venn et al. (2004) Halo stars; open circles, Reddy et al. (2006), mainly thick disk stars. Huge open symbols refer to mean values for globular clusters: star, Palomar 12 (4 stars, Cohen 2004); square M 54 (5 stars, Brown et al. 1999); pentagon, Ruprecht 106 (2 stars, Brown et al. 1997). Ru 106 is included due to its low $[\alpha / \mathrm{Fe}],[\mathrm{Ni} / \mathrm{Fe}]$ ratios, and its high radial velocity, hinting at an extragalactic origin, although its association with Sgr dSph is unlikely (see Pritzl et al. 2005).

comparison. This allows us to estimate the impact of systematic uncertainties in the model parameters. Star \#656 was the one with the largest temperature variation in the sample $\left(\Delta T_{\text {eff }}=\right.$ $-396 \mathrm{~K}, \Delta \log g=-0.4, \Delta \xi=-0.15 \mathrm{~km} \mathrm{~s}^{-1}$, new - old). Despite such a large variation in the parameters, $[\mathrm{X} / \mathrm{Fe}]$ values show remarkably small changes.

\subsection{Comparison with other results}

The only high resolution abundance analysis in the Sgr dSph outside those of our group is the one presented in McWilliam et al. (2003a,b) and McWilliam \& Smecker-Hane (2005a,b). The authors analyze a sample of $14 \mathrm{Sgr} \mathrm{dSph}$ main body giants with higher luminosity and lower temperatures than the ones presented in this work. The bulk of their sample lies in the same metallicity range we explore here, while three stars lie below $[\mathrm{Fe} / \mathrm{H}]=-1$. Despite the difference in stellar parameters and in the details of the analysis, the results coincide for the most part: $\mathrm{Al}, \mathrm{Na}$, and $\mathrm{Cu}$ undersolar ratios are detected, as well as a positive $[\mathrm{La} / \mathrm{Fe}]$, increasing with the metallicity. The authors derive slightly higher $[\alpha / \mathrm{Fe}]$ ratios than we do, but not by a significant amount. The undersolar $[\mathrm{Mn} / \mathrm{Fe}]$ ratio detected in McWilliam et al. $(2003 \mathrm{a}, \mathrm{b})$ is also confirmed by our results, but the lack in our sample of stars below $[\mathrm{Fe} / \mathrm{H}]=-1$ does not allow us to also confirm a trend towards increasing $[\mathrm{Mn} / \mathrm{Fe}]$ with metallicity. In McWilliam \& Smecker-Hane (2005a), the bulk of the analyzed stars show $[\mathrm{Cu} / \mathrm{Fe}] \sim-0.5$, coincident with what we find. This is interesting since a different $\mathrm{Cu}$ I feature is sampled with respect to the one we use; however, four stars show significantly higher $\mathrm{Cu}$ content $([\mathrm{Cu} / \mathrm{Fe}] \sim 0.3)$, something we do not detect.

The composition of three of our 5 Ter 7 giants (\#1282,\#1665 and \#1708) has been studied by Tautvaišienè et al. (2004). We have already compared our results with the ones of Tautvaišienè et al. (2004) in Paper III regarding atmosphere parameters, iron, and $\alpha$ element abundances. While many of the overall trends match (undersolar $\mathrm{Na}$ and $\mathrm{Al}$, oversolar $\mathrm{La}$, etc.), some of the other elements are at odd with our results ( $\mathrm{Sc}, \mathrm{V}, \mathrm{Mn}, \mathrm{Co}, \mathrm{Cu}$, among others). As already stated in Paper III, the atmosphere parameters are fully consistent between us and Tautvaišienè et al. (2004), thus these discrepancies should be due to differences in the line data employed. Nevertheless, since Tautvaišienè et al. (2004) perform a differential analysis, the comparison becomes quite difficult.

\subsection{The long lost Pal 12}

In Figs. 2 through 5, we also show the average value of Palomar 12 stars' abundances from Cohen (2004). By comparing these results against the limited sample of Sgr dSph abundances available at that time, Cohen (2004) already deduced that the strong similarities between the two objects pointed towards the formation of Palomar 12 within the Sgr dSph system. With the present work, the resemblance between Pal 12 and Sgr dSph appears even more clearly, since Pal 12 displays precisely the same "chemical signature" as encountered within Sgr dSph even in its most pronounced anomalies, such as the strong $\mathrm{Na}, \mathrm{Al}$, $\mathrm{Co}, \mathrm{Ni}, \mathrm{Cu}$, and $\mathrm{Zn}$ underabundances. This leads to two main 


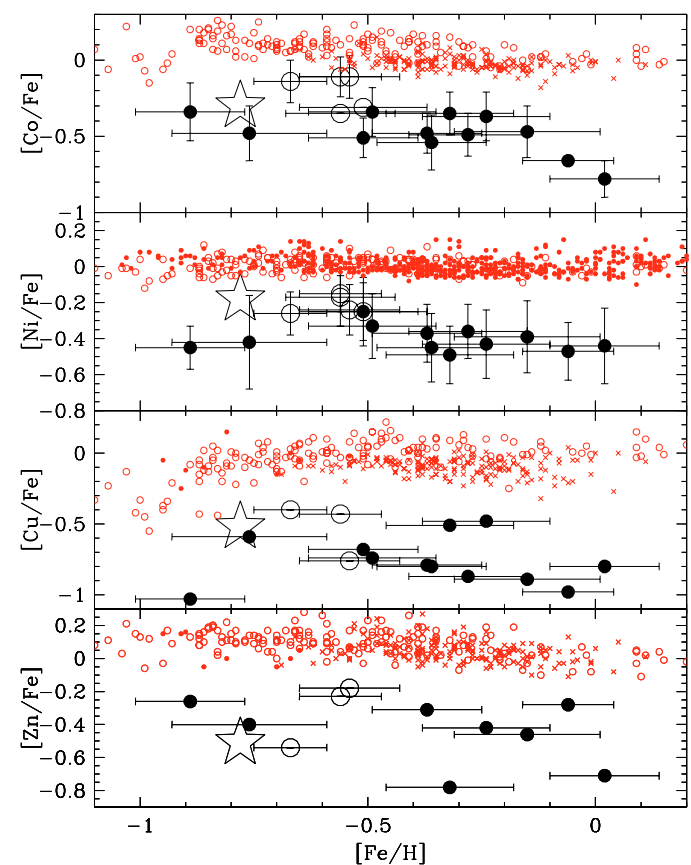

Fig. 4. $[\mathrm{Co} / \mathrm{Fe}],[\mathrm{Ni} / \mathrm{Fe}],[\mathrm{Cu} / \mathrm{Fe}]$, and $[\mathrm{Zn} / \mathrm{Fe}]$ plotted against $[\mathrm{Fe} / \mathrm{H}]$. The symbols are the same as in Fig. 2, except for small filled circles, which indicate the Venn et al. (2004) sample for $\mathrm{Ni}$ and the Bihain et al. (2004) sample for $\mathrm{Cu}$ and $\mathrm{Zn}$.

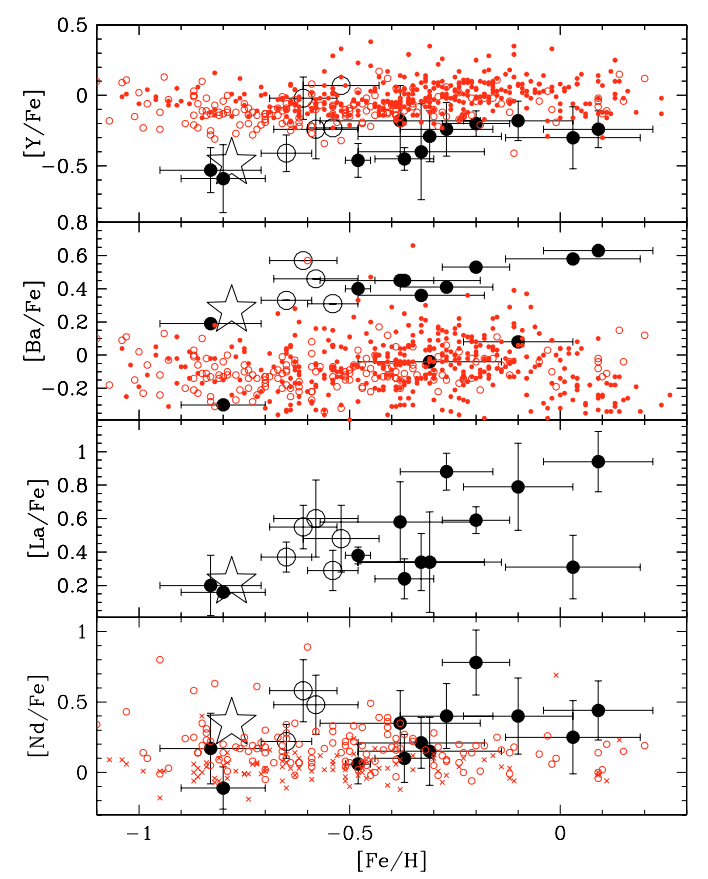

Fig. 5. $[\mathrm{Y} / \mathrm{Fe}],[\mathrm{Ba} / \mathrm{Fe}],[\mathrm{La} / \mathrm{Fe}]$, and $[\mathrm{Nd} / \mathrm{Fe}]$ plotted against $[\mathrm{Fe} / \mathrm{H}]$. Same symbols as in Fig. 2.

results: first, the origin of Pal 12 within the Sgr $\mathrm{SPh}$ can be considered as finally established. There is no known trace of chemically similar populations inside the MW. It can be hypothesized that another (now destroyed) dwarf galaxy may have dropped Pal 12 within the Halo, but such an object should have been a sort of "twin" of Sgr dSph from an evolutionary point of view. The proximity of Pal 12 to the Sgr dSph stream in parameter space is also well known. The second, possibly more intriguing, result is that looking for a Sgr-like "signature" in the chemistry of
Table 3. Variations in the derived abundances $[\mathrm{X} / \mathrm{Fe}]$ and $[\mathrm{Fe} / \mathrm{H}]$ for star \# 656 due to the change in atmospheric parameters between this work and Papers I, II.

\begin{tabular}{llllr}
\hline \hline Ion & $\begin{array}{l}A(\mathrm{X}) \\
\text { Papers I/II }\end{array}$ & $\begin{array}{l}A(\mathrm{X}) \\
\text { this work }\end{array}$ & $\begin{array}{l}\Delta A(\mathrm{X}) \\
\text { new-old }\end{array}$ & $\begin{array}{r}\Delta[\mathrm{X} / \mathrm{Fe}] \\
\text { new-old }\end{array}$ \\
\hline $\mathrm{O}$ I & 8.50 & 8.15 & -0.35 & -0.15 \\
$\mathrm{Na} \mathrm{I}^{a}$ & 5.80 & 5.62 & -0.18 & 0.02 \\
$\mathrm{Mg}$ I & 7.10 & 6.99 & -0.11 & 0.09 \\
$\mathrm{Al}$ I & - & - & - & - \\
$\mathrm{Si}$ I & 7.31 & 7.25 & -0.06 & 0.14 \\
$\mathrm{Ca}$ I & 6.09 & 5.96 & -0.13 & 0.07 \\
$\mathrm{Sc}$ II & 2.55 & 2.34 & -0.21 & 0.06 \\
$\mathrm{Ti}$ I & 4.78 & 4.44 & -0.34 & -0.14 \\
$\mathrm{~V}$ I & 3.80 & 3.38 & -0.42 & -0.22 \\
$\mathrm{Cr}$ II & 5.55 & 5.45 & -0.10 & 0.17 \\
$\mathrm{Mn}$ I & 5.08 & 4.75 & -0.33 & -0.13 \\
$\mathrm{Fe}$ I & 7.33 & 7.13 & -0.20 & - \\
$\mathrm{Fe}$ II & 7.39 & 7.12 & -0.27 & -0.07 \\
$\mathrm{Co}$ I & 4.59 & 4.07 & -0.52 & -0.32 \\
$\mathrm{Ni}$ I & 5.77 & 5.51 & -0.26 & -0.06 \\
$\mathrm{Cu}$ I & 3.44 & 3.05 & -0.39 & -0.19 \\
$\mathrm{Zn}$ I & 3.94 & 3.92 & -0.02 & 0.17 \\
$\mathrm{Y}$ II & 1.93 & 1.68 & -0.25 & 0.02 \\
$\mathrm{Ba}$ II & 2.28 & 2.20 & -0.08 & 0.18 \\
$\mathrm{La}$ II & 1.63 & 1.33 & -0.30 & -0.03 \\
$\mathrm{Ce}$ II & 1.65 & 1.29 & -0.36 & -0.09 \\
$\mathrm{Nd}$ II & 1.86 & 1.47 & -0.39 & -0.12 \\
\hline
\end{tabular}

${ }^{a} \mathrm{Na}$ abundances are listed here without the NLTE correction used elsewhere.

a stellar population is a very effective tool when searching for Sgr dSph debris within the MW, at least at not too low a metallicity. Many of the Sgr dSph chemical oddities appear at any metallicity within the explored range, but what would happen to them at, say, $[\mathrm{Fe} / \mathrm{H}]=-2$ cannot be safely inferred. The trend in $\alpha$ elements seems to resemble the one in MW populations below $[\mathrm{Fe} / \mathrm{H}]=-1$ (see Fig. 3), while $[\mathrm{Cu} / \mathrm{Fe}]$ is known to drop below $[\mathrm{Fe} / \mathrm{H}]=-1$ in the MW. Further analysis of low-metallicity Sgr dSph stars is needed to clarify this.

\section{Discussion and conclusions}

From the observational point of view, our results can be summarized as follows:

1. The chemical composition of the main population of Sgr dSph is significantly at odds with the one observed within the MW. Many elements (Na, Al, Sc, $\alpha$ elements, Co, $\mathrm{Ni}, \mathrm{Cu}, \mathrm{Zn}$, etc.) show significant underabundances when compared to MW stars with a similar iron content. Such chemical oddities are replicated exactly within the associated globular cluster Terzan 7 .

2. Even more interesting, precisely the same "chemical signature" is displayed by Palomar 12. This, as already noted by Cohen (2004), essentially proves that this globular cluster originated within the Sgr dSph system, and was subsequently stripped by the MW.

From the phenomenological point of view, this has one main consequence: chemical composition should allow us to also distinguish other stellar populations that were stripped from Sgr dSph and added to the MW, at least for metallicities above $[\mathrm{Fe} / \mathrm{H}]=-1$. For lower metallicities, the survival of many of the chemical "markers" should be confirmed, but may be inferred 
Table 4. Absolute abundances, assumed solar abundances, [Fe/H], [X/Fe] values and associated errors for Sgr dSph main body stars \#432 to \#716.

\begin{tabular}{|c|c|c|c|c|c|c|c|c|c|c|c|c|c|}
\hline & \multirow{2}{*}{$\begin{array}{l}\text { Sun } \\
A(\mathrm{X})\end{array}$} & \multirow{2}{*}{$\begin{array}{l}432 \\
A(\mathrm{X})\end{array}$} & \multirow[b]{2}{*}{$\sigma$} & \multicolumn{2}{|l|}{628} & \multicolumn{2}{|l|}{635} & \multicolumn{2}{|l|}{656} & \multicolumn{2}{|l|}{709} & \multicolumn{2}{|l|}{716} \\
\hline & & & & $A(\mathrm{X})$ & $\sigma$ & $A(\mathrm{X})$ & $\sigma$ & $A(\mathrm{X})$ & $\sigma$ & $A(\mathrm{X})$ & $\sigma$ & $A(\mathrm{X})$ & $\sigma$ \\
\hline OI & 8.72 & 8.03 & - & 8.35 & - & 8.33 & - & 8.15 & - & 8.50 & - & 8.54 & - \\
\hline $\mathrm{NaI}$ & 6.33 & - & - & 5.81 & 0.08 & 5.40 & 0.03 & 5.74 & 0.08 & 6.03 & 0.13 & 5.83 & 0.16 \\
\hline $\operatorname{Mg} I_{I}$ & 7.58 & 6.64 & 0.01 & 7.16 & 0.12 & 7.10 & 0.13 & 6.99 & 0.04 & 7.21 & 0.12 & 7.13 & 0.02 \\
\hline $\mathrm{Al}$ I & 6.47 & - & - & - & - & - & - & - & - & - & - & 5.61 & - \\
\hline Si I & 7.55 & 6.66 & 0.17 & 7.30 & 0.10 & 7.12 & 0.11 & 7.25 & 0.08 & 7.37 & 0.14 & 7.39 & 0.15 \\
\hline $\mathrm{Ca}$ I & 6.36 & 5.48 & 0.08 & 5.83 & 0.15 & 5.80 & 0.09 & 5.96 & 0.18 & 6.13 & 0.16 & 5.97 & 0.12 \\
\hline Sc II & 3.17 & 2.04 & - & 2.20 & - & 2.45 & - & 2.34 & - & 2.72 & - & 2.58 & - \\
\hline Ti I & 5.02 & 4.01 & 0.08 & 4.44 & 0.15 & 4.13 & 0.14 & 4.44 & 0.12 & 4.59 & 0.21 & 4.38 & 0.12 \\
\hline$V_{\text {I }}$ & 4.00 & - & - & 3.35 & 0.16 & 3.06 & 0.08 & 3.38 & 0.04 & 3.47 & 0.15 & 3.52 & 0.03 \\
\hline Cr II & 5.67 & - & - & - & - & 5.31 & - & 5.45 & - & 5.64 & - & 5.78 & - \\
\hline Mn I & 5.39 & 4.20 & - & 4.83 & - & 4.60 & - & 4.75 & - & 5.03 & - & 4.94 & - \\
\hline $\mathrm{Fe} I$ & 7.50 & 6.61 & 0.12 & 7.22 & 0.13 & 7.14 & 0.12 & 7.13 & 0.12 & 7.52 & 0.12 & 7.35 & 0.16 \\
\hline Fe II & 7.50 & 6.67 & 0.12 & 7.23 & 0.11 & 7.19 & 0.17 & 7.12 & 0.19 & 7.59 & 0.13 & 7.40 & 0.13 \\
\hline Co I & 4.92 & 3.69 & 0.15 & 4.15 & 0.06 & 4.02 & 0.13 & 4.07 & 0.06 & 4.16 & 0.02 & 4.30 & 0.06 \\
\hline $\mathrm{Ni} I$ & 6.25 & 4.91 & 0.01 & 5.61 & 0.08 & 5.44 & 0.15 & 5.51 & 0.10 & 5.83 & 0.17 & 5.71 & 0.12 \\
\hline $\mathrm{Cu} \mathrm{I}$ & 4.21 & 2.29 & - & 3.06 & - & 3.05 & - & 3.05 & - & 3.43 & - & 3.17 & - \\
\hline $\mathrm{ZnI}$ & 4.60 & 3.45 & - & - & - & - & - & 3.92 & - & 3.91 & - & 3.99 & - \\
\hline Y II & 2.24 & 0.88 & 0.11 & 1.73 & 0.15 & 1.64 & 0.05 & 1.68 & 0.16 & 2.09 & 0.03 & 1.96 & 0.05 \\
\hline Ba II & 2.13 & 1.49 & - & 2.27 & - & 1.78 & - & 2.20 & - & 2.85 & - & 2.11 & - \\
\hline La II & 1.13 & 0.50 & 0.14 & 1.74 & 0.02 & 1.16 & 0.25 & 1.33 & 0.15 & 2.16 & 0.12 & 1.82 & 0.22 \\
\hline Ce II & 1.58 & 0.78 & - & 1.58 & 0.02 & 1.48 & 0.09 & 1.29 & 0.06 & 2.11 & 0.21 & 1.89 & 0.13 \\
\hline \multirow[t]{3}{*}{$\mathrm{Nd}$ II } & 1.50 & 0.84 & 0.22 & 1.63 & 0.20 & 1.34 & 0.17 & 1.47 & 0.13 & 2.03 & 0.17 & 1.80 & 0.24 \\
\hline & & 432 & & 628 & & 635 & & 656 & & 709 & & 716 & \\
\hline & & {$[\mathrm{X} / \mathrm{Fe}]$} & $\sigma$ & {$[\mathrm{X} / \mathrm{Fe}]$} & $\sigma$ & {$[\mathrm{X} / \mathrm{Fe}]$} & $\sigma$ & {$[\mathrm{X} / \mathrm{Fe}]$} & $\sigma$ & {$[\mathrm{X} / \mathrm{Fe}]$} & $\sigma$ & {$[\mathrm{X} / \mathrm{Fe}]$} & $\sigma$ \\
\hline$[\mathrm{O} \mathrm{I} / \mathrm{Fe} \mathrm{II}]$ & & 0.14 & - & -0.10 & - & -0.08 & - & -0.19 & - & -0.31 & - & -0.08 & - \\
\hline $\mathrm{NaI}$ & & - & - & -0.24 & 0.15 & -0.57 & 0.12 & -0.22 & 0.14 & -0.32 & 0.18 & -0.35 & 0.23 \\
\hline Mg I & & -0.05 & 0.12 & -0.14 & 0.18 & -0.12 & 0.18 & -0.22 & 0.13 & -0.39 & 0.17 & -0.30 & 0.16 \\
\hline $\mathrm{Al}$ I & & - & - & - & - & - & - & - & - & - & - & -0.71 & - \\
\hline Si I & & 0.00 & 0.21 & 0.03 & 0.16 & -0.07 & 0.16 & 0.07 & 0.14 & -0.20 & 0.18 & -0.01 & 0.22 \\
\hline $\mathrm{Ca}$ I & & 0.01 & 0.14 & -0.25 & 0.20 & -0.20 & 0.15 & -0.03 & 0.22 & -0.25 & 0.20 & -0.24 & 0.20 \\
\hline Sc II & & -0.30 & - & -0.70 & - & -0.41 & - & -0.45 & - & -0.54 & - & -0.49 & - \\
\hline Ti I & & -0.12 & 0.14 & -0.30 & 0.20 & -0.53 & 0.18 & -0.21 & 0.17 & -0.45 & 0.24 & -0.49 & 0.20 \\
\hline VI & & - & - & -0.37 & 0.21 & -0.58 & 0.14 & -0.25 & 0.13 & -0.55 & 0.19 & -0.33 & 0.16 \\
\hline CrII & & - & - & - & - & -0.05 & - & 0.16 & - & -0.12 & - & 0.21 & - \\
\hline Mn I & & -0.30 & - & -0.28 & - & -0.43 & - & -0.27 & - & -0.38 & - & -0.30 & - \\
\hline$[\mathrm{Fe} \mathrm{I} / \mathrm{H}]$ & & -0.89 & 0.12 & -0.28 & 0.13 & -0.36 & 0.12 & -0.37 & 0.12 & 0.02 & 0.12 & -0.15 & 0.16 \\
\hline$[\mathrm{Fe} \mathrm{II} / \mathrm{Fe} \mathrm{I}]$ & & 0.06 & 0.17 & 0.01 & 0.17 & 0.05 & 0.21 & -0.01 & 0.22 & 0.07 & 0.18 & 0.16 & 0.05 \\
\hline Co I & & -0.34 & 0.19 & -0.49 & 0.14 & -0.54 & 0.18 & -0.48 & 0.13 & -0.78 & 0.12 & -0.47 & 0.17 \\
\hline Ni I & & -0.45 & 0.12 & -0.36 & 0.15 & -0.45 & 0.19 & -0.37 & 0.16 & -0.44 & 0.21 & -0.39 & 0.20 \\
\hline $\mathrm{Cu} \mathrm{I}$ & & -1.03 & - & -0.87 & - & -0.80 & - & -0.79 & - & -0.80 & - & -0.89 & - \\
\hline $\mathrm{ZnI}$ & & -0.26 & - & - & - & - & - & -0.31 & - & -0.71 & - & -0.46 & - \\
\hline Y II & & -0.53 & 0.16 & -0.24 & 0.19 & -0.29 & 0.18 & -0.18 & 0.25 & -0.24 & 0.13 & -0.18 & 0.14 \\
\hline Ba II & & 0.19 & - & 0.41 & - & -0.04 & - & 0.45 & - & 0.63 & - & 0.08 & - \\
\hline La II & & 0.20 & 0.18 & 0.88 & 0.11 & 0.34 & 0.30 & 0.58 & 0.24 & 0.94 & 0.18 & 0.79 & 0.26 \\
\hline Ce II & & 0.03 & - & 0.27 & 0.11 & 0.21 & 0.19 & 0.09 & 0.20 & 0.44 & 0.25 & 0.41 & 0.18 \\
\hline Nd II & & 0.17 & 0.25 & 0.40 & 0.23 & 0.15 & 0.24 & 0.35 & 0.23 & 0.44 & 0.21 & 0.40 & 0.27 \\
\hline
\end{tabular}

in many cases by the absence of metallicity trends within them (e.g. $\mathrm{Na}, \mathrm{Ni}, \mathrm{Zn})$.

Nevertheless, it should be taken into account that no population with Sgr-like characteristics has been found within the MW. Recently, Chou et al. (2006) has claimed detection of metallicity gradients within the Sgr dSph stream, in the sense of higher metallicities in those populations stripped more recently from the galaxy. From this finding, we can infer that a significant chemical evolution has taken place in the galaxy since the most distant stream stars were stripped, or (more likely) that the outermost parts of Sgr dSph (the first to be stripped) were significantly more metal poor than the nucleus. A combination of both scenarios is also possible. In all these cases, the "chemical signature" we detect in the main body of Sgr dSph may well be much weaker, or even undetectable in the Halo at metallicities below
$[\mathrm{Fe} / \mathrm{H}]=-1$. Conversely, Palomar 12 stands as an unmistakable example of how evident this signature can be in more chemically evolved stars.

It appears a much harder task to decrypt the meaning of these abundances in terms of the chemical history of Sgr dSph, and we will not attempt to go more deeply in this direction in the present paper. Sgr dSph appears to have experienced a very long star formation and chemical evolution. The lowest metallicity observed within the galaxy is about $[\mathrm{Fe} / \mathrm{H}]=-3$ (Zaggia et al. 2004), while the associated globulars (M 54, Ter 7, Ter 8, Arp 2, $\mathrm{Pal} 12)$ are dispersed between $[\mathrm{Fe} / \mathrm{H}]=-2$ and $[\mathrm{Fe} / \mathrm{H}]=-0.6$. At least the last few GYr of the galaxy evolution have taken place within the strong MW tidal field, which should have heavily influenced both its star formation and its capability of retaining the chemically enriched gas expelled by SN explosions. This may 
Table 5. Same as in Table 4 for stars \#717 to \#927.

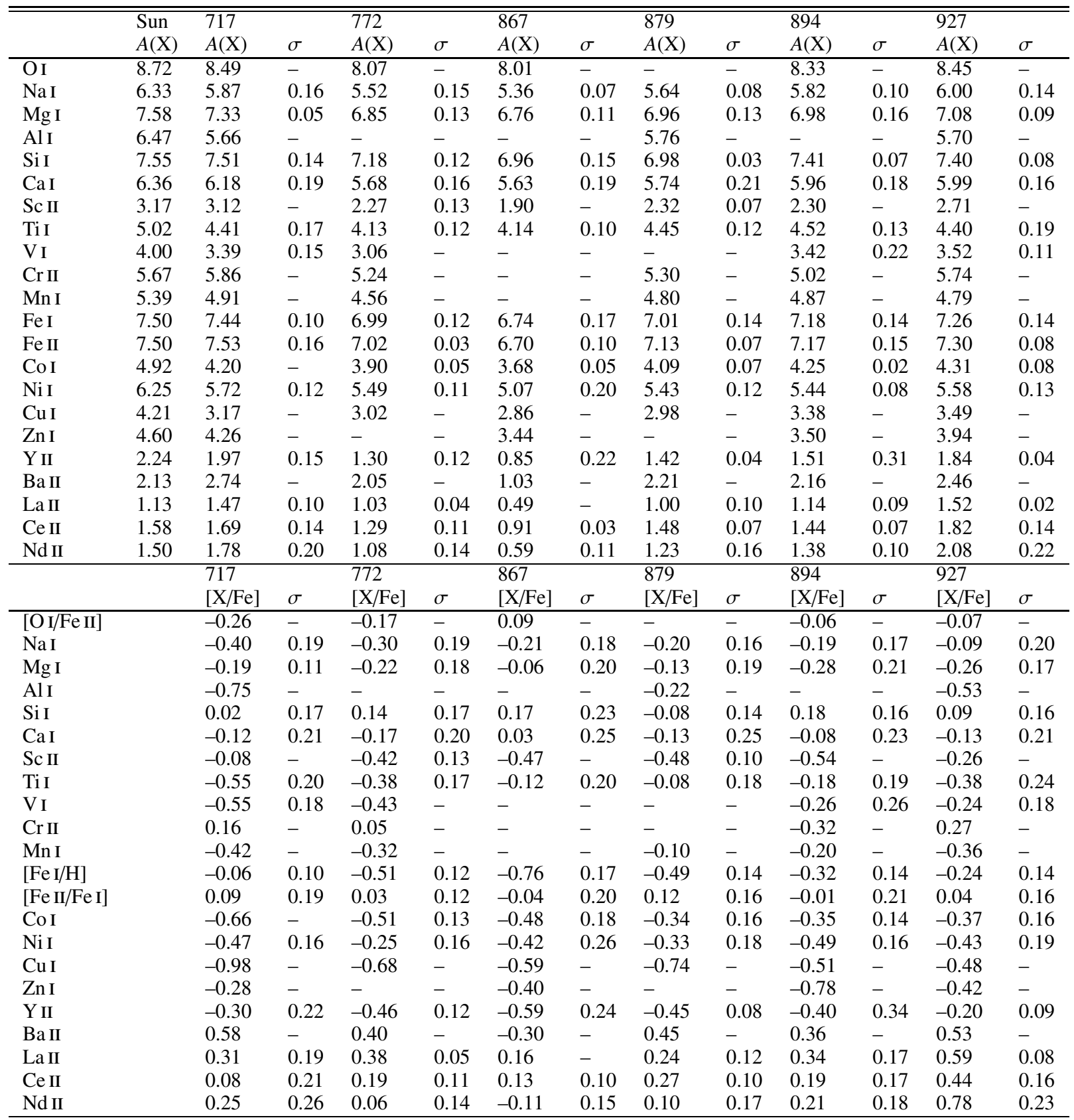

help explain both the high mean metallicity of Sgr dSph and its scarce gas content in spite of having formed stars until a relatively recent past (probably a couple of GYr, see Paper II). At the same time, this may call for past (pre-interaction) Sgr dSph being a relatively large, star-forming, gas-rich object with a nucleated structure (Monaco et al. 2005b) and a rich set of globular clusters.

The detailed abundance ratios we present need to be interpreted in the framework of a detailed chemical evolution model taking galactic winds into account (such as in Lanfranchi \& Matteucci 2003, 2004; Lanfranchi et al. 2006a,b). Galactic winds appear to play a key role in the evolution of small systems such as dSph, where they develop thanks to the relatively weak gravitational field these small galaxies create. Nevertheless, they still are more inferred than modeled, due to the big difficulty with the hydrodynamical calculations that would be needed. In the particular case of Sgr dSph, the influence of the MW tidal field has very likely favored wind formation, an effect that has been most likely time dependent, since Sgr dSph orbit should have degraded with time.

Aside from all these issues, we want to stress how these abundances should not be taken as plainly representative of Sgr dSph "as a whole". This not only comes from the narrow range in metallicity covered, but also from the small angular area from which they have been collected. Chou et al. (2006) findings point towards a scenario in which pre-interaction Sgr dSph may have shown strong metallicity gradients, or maybe even an incomplete chemical mixing. Especially at low metallicities, chemical enrichment may have been locally influenced by a small number of SN II, due to the low total mass of the galaxy. 
Table 6. Same as in Table 4 but now for Terzan 7 stars.

\begin{tabular}{|c|c|c|c|c|c|c|c|c|c|c|c|}
\hline & Sun & 1272 & & 1282 & & 1515 & & 1665 & & 1708 & \\
\hline & $A(\mathrm{X})$ & $A(\mathrm{X})$ & $\sigma$ & $A(\mathrm{X})$ & $\sigma$ & $A(\mathrm{X})$ & $\sigma$ & $A(\mathrm{X})$ & $\sigma$ & $A(\mathrm{X})$ & $\sigma$ \\
\hline$\overline{\mathrm{OI}}$ & 8.72 & 8.17 & - & 8.36 & - & - & - & 8.43 & - & 8.34 & - \\
\hline $\mathrm{NaI}$ & 6.33 & 5.49 & 0.02 & 5.48 & 0.06 & 5.42 & 0.04 & 5.49 & 0.07 & 5.32 & 0.04 \\
\hline $\mathrm{Mg}_{\mathrm{I}}$ & 7.58 & 6.88 & 0.13 & 6.88 & 0.05 & 6.99 & 0.09 & 6.93 & 0.12 & 6.84 & 0.08 \\
\hline $\mathrm{Al} \mathrm{I}$ & 6.47 & 5.55 & 0.11 & 5.47 & 0.01 & 5.66 & - & 5.43 & 0.02 & 5.43 & - \\
\hline Si I & 7.55 & 6.91 & 0.16 & 7.06 & 0.11 & 7.14 & 0.05 & 7.00 & 0.12 & 6.98 & 0.20 \\
\hline S I & 7.21 & - & - & 6.62 & 0.04 & - & - & 6.62 & 0.04 & 6.59 & 0.04 \\
\hline $\mathrm{CaI}$ & 6.36 & 5.88 & 0.09 & 5.74 & 0.09 & 5.85 & 0.10 & 5.79 & 0.11 & 5.65 & 0.05 \\
\hline Sc II & 3.17 & 2.15 & 0.01 & 2.36 & 0.04 & 2.42 & 0.04 & 2.30 & 0.04 & 2.29 & 0.15 \\
\hline Ti I & 5.02 & 4.47 & 0.09 & 4.50 & 0.13 & 4.52 & 0.07 & 4.59 & 0.21 & 4.39 & 0.11 \\
\hline $\mathrm{V}_{\mathrm{I}}$ & 4.00 & 3.35 & 0.16 & 3.35 & 0.03 & 3.43 & 0.04 & 3.43 & 0.07 & 3.20 & 0.03 \\
\hline $\mathrm{Cr} \mathrm{I}$ & 5.67 & 4.99 & 0.02 & 5.05 & 0.04 & 5.02 & 0.04 & 5.17 & 0.08 & 5.03 & 0.09 \\
\hline Mn I & 5.39 & 4.47 & - & 4.58 & - & 4.45 & - & - & - & - & - \\
\hline $\mathrm{Fe} I$ & 7.50 & 6.83 & 0.08 & 6.96 & 0.11 & 6.94 & 0.09 & 6.99 & 0.14 & 6.94 & 0.12 \\
\hline Fe II & 7.50 & 6.85 & 0.06 & 6.92 & 0.10 & 6.89 & 0.08 & 6.98 & 0.09 & 6.96 & 0.06 \\
\hline CoI & 4.92 & 4.11 & 0.12 & 4.27 & 0.09 & 4.25 & 0.09 & 4.10 & - & 4.01 & - \\
\hline Ni I & 6.25 & 5.32 & 0.09 & 5.47 & 0.08 & 5.54 & 0.05 & 5.49 & 0.13 & 5.52 & 0.11 \\
\hline $\mathrm{Cu} \mathrm{I}$ & 4.21 & 3.14 & - & 2.91 & - & 3.22 & - & - & - & - & - \\
\hline Zn I & 4.60 & 3.39 & - & 3.88 & - & 3.81 & - & - & - & - & - \\
\hline Y II & 2.24 & 1.18 & 0.11 & 1.42 & 0.19 & 1.61 & 0.13 & 1.79 & - & 1.47 & - \\
\hline Ba II & 2.13 & 1.81 & - & 2.01 & - & 2.09 & - & - & - & 1.90 & - \\
\hline La II & 1.13 & 0.85 & 0.07 & 1.15 & 0.21 & 1.07 & 0.10 & 1.09 & 0.18 & 0.88 & 0.10 \\
\hline Ce II & 1.58 & 1.03 & 0.11 & 1.27 & 0.14 & 1.51 & 0.11 & - & - & - & - \\
\hline \multirow[t]{2}{*}{$\mathrm{Nd}$ II } & 1.50 & 1.07 & 0.10 & 1.40 & 0.19 & 1.47 & 0.21 & - & - & - & - \\
\hline & & $\begin{array}{l}1272 \\
{[\mathrm{X} / \mathrm{Fe}]}\end{array}$ & $\sigma$ & $\begin{array}{l}1282 \\
{[\mathrm{X} / \mathrm{Fe}]}\end{array}$ & $\sigma$ & $\begin{array}{l}1515 \\
{[\mathrm{X} / \mathrm{Fe}]}\end{array}$ & $\sigma$ & $\begin{array}{l}1665 \\
{[\mathrm{X} / \mathrm{Fe}]}\end{array}$ & $\sigma$ & $\begin{array}{l}1708 \\
{[\mathrm{X} / \mathrm{Fe}]}\end{array}$ & $\sigma$ \\
\hline$[\mathrm{OI} / \mathrm{Fe} \mathrm{II}]$ & & 0.10 & - & 0.22 & - & - & - & 0.23 & - & 0.16 & - \\
\hline $\mathrm{NaI}$ & & -0.17 & 0.08 & -0.31 & 0.13 & -0.35 & 0.10 & -0.33 & 0.16 & -0.45 & 0.13 \\
\hline Mg I & & -0.03 & 0.15 & -0.16 & 0.12 & -0.03 & 0.13 & -0.14 & 0.18 & -0.18 & 0.14 \\
\hline $\mathrm{Al} \mathrm{I}$ & & -0.25 & 0.14 & -0.46 & 0.11 & -0.25 & - & -0.53 & 0.14 & -0.48 & - \\
\hline Si I & & 0.03 & 0.18 & 0.05 & 0.16 & 0.15 & 0.10 & -0.04 & 0.18 & -0.01 & 0.23 \\
\hline S I & & - & - & -0.05 & 0.12 & - & - & -0.08 & 0.15 & -0.06 & 0.13 \\
\hline $\mathrm{CaI}$ & & 0.19 & 0.12 & -0.08 & 0.14 & 0.05 & 0.13 & -0.06 & 0.18 & -0.15 & 0.13 \\
\hline Sc II & & -0.37 & 0.06 & -0.23 & 0.11 & -0.14 & 0.09 & -0.35 & 0.10 & -0.34 & 0.16 \\
\hline Ti I & & 0.12 & 0.12 & 0.02 & 0.17 & 0.06 & 0.11 & 0.08 & 0.25 & -0.07 & 0.16 \\
\hline V I & & 0.02 & 0.18 & -0.11 & 0.11 & -0.01 & 0.10 & -0.06 & 0.16 & -0.24 & 0.12 \\
\hline CrI & & -0.01 & 0.08 & -0.08 & 0.12 & -0.09 & 0.10 & 0.01 & 0.16 & -0.08 & 0.15 \\
\hline Mn I & & -0.25 & - & -0.27 & - & -0.38 & - & - & - & - & - \\
\hline$[\mathrm{Fe} \mathrm{I} / \mathrm{H}]$ & & -0.67 & 0.08 & -0.54 & 0.11 & -0.56 & 0.09 & -0.51 & 0.14 & -0.56 & 0.12 \\
\hline$[\mathrm{Fe}$ II $/ \mathrm{Fe} \mathrm{I}]$ & & 0.02 & 0.10 & -0.04 & 0.15 & -0.05 & 0.12 & -0.01 & 0.17 & 0.02 & 0.13 \\
\hline Co I & & -0.14 & 0.14 & -0.11 & 0.14 & -0.11 & 0.13 & -0.31 & - & -0.35 & - \\
\hline Ni I & & -0.26 & 0.12 & -0.24 & 0.14 & -0.15 & 0.10 & -0.25 & 0.19 & -0.17 & 0.16 \\
\hline $\mathrm{Cu} \mathrm{I}$ & & -0.40 & - & -0.76 & - & -0.43 & - & - & - & - & - \\
\hline Zn I & & -0.54 & - & -0.18 & - & -0.23 & - & - & - & - & - \\
\hline Y II & & -0.41 & 0.13 & -0.24 & 0.21 & -0.02 & 0.15 & 0.07 & - & -0.23 & - \\
\hline Ba II & & 0.33 & - & 0.46 & - & 0.57 & - & - & - & 0.31 & - \\
\hline La II & & 0.37 & 0.09 & 0.60 & 0.23 & 0.55 & 0.13 & 0.48 & 0.20 & 0.29 & 0.12 \\
\hline Ce II & & 0.10 & 0.13 & 0.27 & 0.17 & 0.54 & 0.14 & - & - & - & - \\
\hline Nd II & & 0.22 & 0.12 & 0.48 & 0.21 & 0.58 & 0.22 & - & - & - & - \\
\hline
\end{tabular}

Larger sampling across the galaxy body and the streams are needed, as well as analyses of the more metal poor components, to be able to trace the full story of this fascinating neighbor.

Acknowledgements. L.S., P.B. and S.Z. acknowledge support from the MIUR/PRIN 2004025729_002; L.S. and P.B. are also supported by EU contract MEXT-CT-2004-014265 (CIFIST). This work has made use of NASA's Astrophysics Data System Bibliographical Services and of NIST online database of atomic data.

\section{References}

Alonso, A., Arribas, S., \& Martínez-Roger, C. 1999, A\&AS, 140, 261 Alonso, A., Arribas, S., \& Martínez-Roger, C. 2001, A\&A, 376, 1039 Arnesen, A., Bengtsson, A., Hallin, R., et al. 1977, Phys. Scr., 16, 31 (ABH) Baumueller, D., \& Gehren, T. 1997, A\&A, 325, 1088
Baumueller, D., Butler, K., \& Gehren, T. 1998, A\&A, 338, 637 Belokurov, V., Zucker, D. B., Evans, N. W., et al. 2006, ApJ, 642, L137 Bielski, A. 1975, JQSRT, 15, 463 (BIEL)

Biemont, E., \& Godefroid, M. 1980, A\&A, 84, 361 (BG)

Bihain, G., Israelian, G., Rebolo, R., Bonifacio, P., \& Molaro, P. 2004, A\&A, 423, 777

Bonifacio, P., Hill, V., Molaro, P., et al. 2000, A\&A, 359, 663

Bonifacio, P., Sbordone, L., Marconi, G., Pasquini, L., \& Hill, V. 2004, A\&A, 414,503

Brown, J. A., Wallerstein, G., \& Zucker, D. 1997, AJ, 114, 180

Brown, J. A., Wallerstein, G., \& Gonzalez, G. 1999, AJ, 118, 1245

Burbidge, E. M., Burbidge, G. R., Fowler, W. A., \& Hoyle, F. 1957, Rev. Mod. Phys., 29, 547

Caffau, E., Bonifacio, P., Faraggiana, R., \& Sbordone, L. 2005, A\&A, 436, L9

Castelli, F., \& Kurucz, R. L. 2003, Modelling of Stellar Asmospheres, ed. N. Piskunov et al., poster A20 on the enclosed CD-ROM, Proc. IAU Symp., 210 [arXiv: astro-ph/0405087] 
Chou, M.-Y., Majewski, S. R., Cunha, K., et al. 2006, ArXiv Astrophysics e-prints [arXiv: astro-ph/0605101]

Cohen, J. G. 2004, AJ, 127, 1545

Cowley, C. R., \& Corliss, C. H. 1983, MNRAS, 203, 651 (CC)

Den Hartog, E. A., Lawler, J. E., Sneden, C., \& Cowan, J. J. 2003, ApJS, 148, $543(\mathrm{HA})$

Edvardsson, B., Andersen, J., Gustafsson, B., et al. 1993, A\&A, 275, 101 (ED)

Fuhr, J. R., Martin, G. A., \& Wiese, W. L. 1988, J. Phys. Chem. Ref. Data, 17, Suppl. 4 (FMW)

Garz, T. 1973, A\&A, 26, 471 (GARZ)

Girardi, L., Bertelli, G., Bressan, A., et al. 2002, A\&A, 391, 195

Gratton, R. G., Carretta, E., Eriksson, K., \& Gustafsson, B. 1999, A\&A, 350, 955

Gratton, R. G., Carretta, E., Desidera, S., et al. 2003, A\&A, 406, 131 (GC)

Hannaford, P., Lowe, R. M., Grevesse, N., Biemont, E., \& Whaling, W. 1982, ApJ, 261, 736 (HL)

Hannaford, P., Lowe, R. M., Grevesse, N., \& Noels, A. 1992, A\&A, 259, 301 (H92)

Helmi, A., \& White, S. D. M. 1999, MNRAS, 307, 495

Hill, V., Andrievsky, S., \& Spite, M. 1995, A\&A, 293, 347 (H)

Ibata, R. A., Gilmore, G., \& Irwin, M. J. 1994, Nature, 370, 194

Ibata, R. A., Gilmore, G., \& Irwin, M. J. 1995, MNRAS, 277, 781

Ibata, R. A., Wyse, R. F. G., Gilmore, G., Irwin, M. J., \& Suntzeff, N. B. 1997, AJ, 113, 634

Johansson, S., Litzén, U., Lundberg, H., \& Zhang, Z. 2003, ApJ, 584, L107

Kurucz, R. L. 1988, Trans. IAU, XXB, ed. M. McNally (Dordrecht: Kluwer), 168 (K88)

Kurucz, R. L. 1993, CDROM, 13, 18

Kurucz, R. L. 2005, Mem. Soc. Astron. It. Suppl., 8, 14

Kurucz, R. L., \& Peytremann, E. 1975, SAO Special Report, 362 (KP)

Lanfranchi, G. A., \& Matteucci, F. 2003, MNRAS, 345, 71

Lanfranchi, G. A., \& Matteucci, F. 2004, MNRAS, 351, 1338

Lanfranchi, G. A., Matteucci, F., \& Cescutti, G. 2006a, MNRAS, 365, 477

Lanfranchi, G. A., Matteucci, F., \& Cescutti, G. 2006b, A\&A, 453, 67

Laurent, J., \& Weniger, S. 1970, JQSRT, 10, 315 (LW)

Lawler, J. E., Bonvallet, G., \& Sneden, C. 2001, ApJ, 556, 452 (LA)

Layden, A. C., \& Sarajedini, A. 2000, AJ, 119, 1760

Majewski, S. R., Skrutskie, M. F., Weinberg, M. D., \& Ostheimer, J. C. 2003, ApJ, 599, 1082

Marconi, G., Buonanno, R., Castellani, M., et al. 1998, A\&A, 330, 453
Martin, G. A., Fuhr, J. R., \& Wiese, W. L. 1988, J. Phys. Chem. Ref. Data, 17, Suppl. 3 (MFW)

McWilliam, A., \& Smecker-Hane, T. A. 2005a, ApJ, 622, L29

McWilliam, A., \& Smecker-Hane, T. A. 2005b, Cosmic Abundances as Records of Stellar Evolution and Nucleosynthesis, ASP Conf. Ser., 336, 221

McWilliam, A., Rich, R. M., \& Smecker-Hane, T. A. 2003a, ApJ, 592, L21

McWilliam, A., Rich, R. M., \& Smecker-Hane, T. A. 2003b, ApJ, 593, L145

Meggers, W. F., Corliss, C. H., \& Scribner, B. F. 1975, NBS Monograph, 145 (MC')

Miles, B. M., \& Wiese, W. L. 1969, NBS Technical Note, 474 (NBS for Ba)

Monaco, L., Ferraro, F. R., Bellazzini, M., \& Pancino, E. 2002, ApJ, 578, L47

Monaco, L., Bellazzini, M., Ferraro, F. R., \& Pancino, E. 2004, MNRAS, 353, 874

Monaco, L., Bellazzini, M., Bonifacio, P., et al. 2005a, A\&A, 441, 141

Monaco, L., Bellazzini, M., Ferraro, F. R., \& Pancino, E. 2005b, MNRAS, 356, 1396

Pritzl, B. J., Venn, K. A., \& Irwin, M. 2005, AJ, 130, 2140

Reddy, B. E., Tomkin, J., Lambert, D. L., \& Allende Prieto, C. 2003, MNRAS, 340,304

Reddy, B. E., Lambert, D. L., \& Allende Prieto, C. 2006, MNRAS, 343

Sbordone, L. 2005, Mem. Soc. Astron. It. Suppl., 8, 61

Sbordone, L., Bonifacio, P., Castelli, F., \& Kurucz, R. L. 2004, Mem. Soc. Astron. It. Suppl., 5, 93

Sbordone, L., Bonifacio, P., Marconi, G., Buonanno, R., \& Zaggia, S. 2005, A\&A, 437, 905

Schlegel, D. J., Finkbeiner, D. P., \& Davis, M. 1998, ApJ, 500, 525

Smith, G., \& Raggett, D. S. J. 1981, J. Phys. Atom. Mol. Phys., 14, 4015 (SR)

Shetrone, M. D., Côté, P., \& Sargent, W. L. W. 2001, ApJ, 548, 592

Shetrone, M., Venn, K. A., Tolstoy, E., et al. 2003, AJ, 125, 684

Storey, P. J., \& Zeippen, C. J. 2000, MNRAS, 312, 813 (STZ)

Tautvaišienè, G., Wallerstein, G., Geisler, D., Gonzalez, G., \& Charbonnel, C. 2004, AJ, 127, 373

Venn, K. A., Irwin, M., Shetrone, M. D., et al. 2004, AJ, 128, 1177

Vladilo, G., Sbordone, L., \& Bonifacio, P. 2003, The Local Group as an Astrophysical Laboratory, 107

Wiese, W. L., \& Fuhr, J. R. 1975, J. Phys. Chem. Ref. Data, 4, 263 (NBS for Sc)

Wiese, W. L., Smith, M. W., \& Miles, B. M. 1969, NSRDS-NBS 22 (NBS for $\mathrm{Al}, \mathrm{S}$ and $\mathrm{Ca})$

Zaggia, S., Bonifacio, P., Bellazzini, M., et al. 2004, Mem. Soc. Astron. It. Suppl., 5, 291 